\title{
Telehealth strategy to mitigate the negative psychological impact of the COVID-19 pandemic on type 2 diabetes: A randomized controlled trial
}

\author{
Janine Alessi ${ }^{1,2}$. Giovana Berger de Oliveira ${ }^{3}$. Debora Wilke Franco ${ }^{3}$. Alice Scalzilli Becker ${ }^{3}$. \\ Carolina Padilla Knijnik ${ }^{3}$. Gabriel Luiz Kobe ${ }^{3}$. Bibiana Brino Amaral ${ }^{3}$ - Ariane de Brito ${ }^{1}$ - Beatriz D. Schaan ${ }^{1,4,5}$. \\ Gabriela Heiden Telo ${ }^{2,3,6}$
}

Received: 28 November 2020 / Accepted: 22 February 2021 / Published online: 15 March 2021

(c) Springer-Verlag Italia S.r.l., part of Springer Nature 2021

\begin{abstract}
Aims To assess the impact of teleintervention on mental health parameters in type 2 diabetes patients during the coronavirus disease 2019 (COVID-19) pandemic.

Methods This is a controlled randomized trial for a multidisciplinary telehealth intervention in Southern Brazil, with social distancing measures. Adults aged 18 years or older with previous diagnosis of type 2 diabetes were included in the study. The intervention performed was a set of strategies to help patients stay healthy during the COVID-19 pandemic and included the maintaining of telephone contacts and providing educational materials on issues related to mental health, healthy habits, and diabetes care. The primary outcome was a positive screening for mental health disorders (Self-Reporting Questionnaire) after 16 weeks of intervention. A positive screening for mental health disorders was considered when the survey scored greater than or equal to 7. Secondary outcomes included a positive screening for diabetes-related emotional distress (Problem Areas in Diabetes), eating (Eating Attitudes Test), and sleep disorders (Mini Sleep Questionnaire). Comparisons with $\chi^{2}$ tests for dichotomous outcomes, along with the Mann-Whitney U test, was used for between group analyses.

Results A total of 91 individuals agreed to participate (46 intervention group and 45 control group). There were no differences in demographic and clinical data at baseline. After 16 weeks of follow-up, a positive screening for mental health disorders was found in $37.0 \%$ of participants in the intervention group vs. $57.8 \%$ in the control group $(P=0.04)$. Diabetes-related emotional distress was found in $21.7 \%$ of participants in the intervention group vs. $42.2 \%$ in the control group $(P=0.03)$. No differences were found between groups with regard to eating and sleep disorders.

Conclusion This study demonstrated that maintaining remote connections with health professionals during social distancing and quarantine have the potential to reduce the prevalence of positive screening for mental health disorders and diabetesrelated emotional distress in adults with type 2 diabetes.
\end{abstract}

Keywords COVID-19 pandemic · Diabetes-related distress · Teleintervention · Type 2 diabetes

This article belongs to the topical collection Health Education and Psycho-Social Aspects, managed by Massimo Porta and Marina Trento.

Janine Alessi

janinealessi@gmail.com

Extended author information available on the last page of the article

\section{Introduction}

Since the beginning of the coronavirus disease 2019 (COVID-19) pandemic, diabetes was one factor associated with worse clinical outcomes due to mechanisms not fully understood $[1,2]$. The threat of a potentially serious infection and being part of a high-risk group creates worry and anxiety in patients with diabetes, making them vulnerable to mental health disorders. In addition to concerns about the COVID-19 pandemic and the possibility of a potentially serious infection, patients with diabetes experienced other challenges during the pandemic. Health appointments not 
fully accessible, difficulty in obtaining diabetes medications, and a lack of scientific information about the relationship between diabetes and COVID-19 result in psychological and emotional strain [3]. Even in non-pandemic circumstances, people with diabetes have more mood and anxiety disorders compared to the general population [4-6]. This greater psychological vulnerability was exacerbated during the COVID-19 pandemic [4], reflecting the need to care for the mental health of patients with diabetes.

So far, there is a lack of evidence-based guidelines, as well as a clear intervention plan to safeguard the health of patients with diabetes and mitigate COVID-19 pandemic effects on mental health with social distancing $[7,8]$. Remote interventions and virtual platforms could be potential strategies to allow providers to assist patients in the quarantine period [9]. This study aims to assess the impact of teleinterventions on mental health parameters in those with type 2 diabetes during the COVID-19 pandemic in Brazil. We hypothesize that remote assistance may improve mental health outcomes.

\section{Participants and methods}

\section{Study design}

An open-label and controlled randomized trial was conducted for a telehealth intervention during the COVID-19 pandemic. Electronic medical records were used to identify adults with a previous diagnosis of type 2 diabetes with regular outpatient follow-up in one of two public tertiary care hospitals in Southern Brazil. Study procedures started in April 2020, approximately one month after the national ordinance required social distancing for risk groups in Brazil. The study was done in accordance with the Helsinki Declaration, 2004, approved by the National Scientific Committee (No. 4.059.760). This trial was registered at ClinicalTrials.gov (NCT04344210). Enrollment began on April 14, 2020, and ended on April 29, 2020. This reporting follows the CONSORT statement [10]

\section{Participants}

Inclusion criteria involved: adults aged 18 years or older with a previous diagnosis of type 2 diabetes based on guideline recommendations; a HbA1c evaluation in the laboratory of the study's reference hospital in the three months prior to inclusion; and availability for weekly phone calls during the study. Patients hospitalized at the time of recruitment and those who had some serious limitation preventing the necessary interaction, such as advanced dementia or severe hearing loss, were excluded.

\section{Recruitment procedure}

Potential participants were identified by electronic databases from the main institutions. Patients who met inclusion criteria were randomly selected for a telephone call that included an invitation to participate in this study. Those who agreed provided informed consent electronically, including audio recording.

\section{Allocation}

After providing informed consent to participate in this study, patients were randomly assigned in a 1:1 ratio with an electronic database, generated by the Randomization. com website. Due to the characteristics of the intervention, blinding of participants and researchers was not possible. To minimize potential bias, inclusion in the study and assessment of outcomes were performed by different researchers, who were not involved in the randomization and data analyses.

\section{Intervention characteristics}

The intervention was a set of strategies to help patients stay healthy during the COVID-19 pandemic, which included telephone calls, offering educational skills on healthy lifestyles, and complementing patients' usual clinical care. Remote methods preserved patients with diabetes from unnecessary exposure during social distancing. To develop intervention protocols and provide support for patient demands, a multidisciplinary team of general practitioners, cardiologists, endocrinologists, physical educators, and psychologists was available.

(1) Telehealth intervention: Each participant was randomly assigned to be followed by a same trained researcher, called case manager, responsible for making weekly calls for 16 weeks of follow-up. The calls lasted around 5 to $10 \mathrm{~min}$ and followed a pre-established script to ensure all patients received a similar intervention. Intervention protocols were developed by physicians, physical educators, and psychologists. Each week, a different topic was selected for participants. Topics included issues related to mental health and coping strategies, physical activity, healthy eating habits, and diabetes care. Complete scripts used for the 16 weeks of intervention are available in supplementary material.

(2) Educational materials: Patients with difficulty in a specific area related to mental health or diabetes care received additional digital educational material. Information about healthy eating habits and physical 
exercise adapted to age and physical limitations were offered to all participants.

(3) Clinical care: Intervention sought to complement the usual diabetes care. Adherence to treatment was encouraged on every call. Patients were routinely asked for reports on glycemic controls, following their provider's recommendation. Treatment adjustments were made in severe cases, such as recurrent hypoglycemia and difficulty in contacting health care providers. In those cases, treatment was recommended by an endocrinologist from the study team. If patients had outdated prescriptions and difficulty in contacting the health care providers, renewal was electronically carried out by the researchers. All specific issues that arose during the intervention were discussed with the multidisciplinary team.

\section{Active control group}

At randomization, the active control group received access to a website prepared by the multidisciplinary team. On this site, there were weekly posts about diabetes care, mental health, and lifestyle habits, similar to phone call protocols. Access to the website was free, with no control on the frequency of access. This group did not have direct contact with the researchers during 16 weeks of the study.

\section{Outcome measures}

The original protocol was designed to assess metabolic outcomes, and mental health would be included as secondary outcomes. However, considering the expressive impact of the pandemic on mental health, a new sample calculation was carried out to determine the sample size necessary for mental health as the primary outcome. This entire procedure was performed before the inclusion of participants in the study. Due to the extent of the pandemic in Brazil, which lasted longer than expected, and the limitation of laboratory tests during social distancing, it was impossible to perform the glycemic control and mental health assessment at the same time. So, the primary outcome in this analysis was only about mental health.

Mental health outcomes were assessed using specific questionnaires, applied by trained researchers in telephone calls. To assess the effects of the intervention, all participants were evaluated at two different times: at inclusion in the study (baseline) and after 16 weeks of intervention. Baseline represents the first four weeks of social distancing during the COVID-19 pandemic, while follow-up (evaluated after 16 weeks) represents the results after 20 weeks of social distancing in Brazil. The follow-up for the primary outcome was performed within 14 days, to yield the same pandemic period for all participants.
The primary outcome was a comparison between the two groups of the presence of a positive screening for mental health disorders at the 16-week follow-up period. For this, the Brazilian validated version of the Self Report Questionnaire-20 (SRQ-20) was used. A positive screening for mental health disorders was considered when the survey scored greater than or equal to 7 [11].

Secondary outcomes included a comparison of the 16-week follow-up period between the two groups for a positive screening and for diabetes-related emotional distress, eating disorders, sleep disorders and treatment adherence. Diabetes-related emotional distress was assessed by the Brazilian validated version of the Problem Areas in Diabetes Scale (B-PAID) and considered when the score was greater than or equal to 40 [12]. Eating disorders were assessed by the Brazilian validated version of the Eating Attitudes Test (EAT-26). A positive screening for eating disorders was considered when the score was greater than or equal to 20 [13]. To assess sleep disorders, the Brazilian version of the Mini Sleep Questionnaire (MSQ) was used: a positive screening was considered for a score greater than or equal to 31 [14]. To assess treatment adherence, a BrazilianPortuguese version of the Self-Care Inventory-revised (SCI$\mathrm{R})$ was used, and higher scores represent greater adherence [15].

At the end of the follow-up period, questionnaires were applied for changes that occurred during the COVID-19 pandemic. This assessment included: social distancing, maintenance of work activities, financial difficulties, and medical assistance. An assessment of patients' respiratory symptoms, as well as the presence of COVID-19 confirmed infection was also carried out.

Demographics and clinical data on diabetes, presence of comorbidities, continuous use of medications, and the HbA1c value evaluated by high-performance liquid chromatography were collected from electronic medical records. Information about antidepressant or anxiolytic drugs and a previous diagnosis of psychiatric disorders were found in electronic medical records and checked with patients.

\section{Statistical methods}

\section{Power estimations for primary outcome:}

The initial protocol was designed for two primary outcomes: mental health and HbA1c levels. To detect a difference in $\mathrm{HbA} 1 \mathrm{c}$ values of moderate effect size between groups with a power of $80 \%$, a significance level of 0.05 , and a total of 84 participants was necessary. For the mental health primary outcome, a total of 78 participants were required to detect a moderate effect size $(0.5)$ for a minimum difference of 5.8 points between the groups in mental health disorders (16). Considering estimated withdrawal from the study, the 
sample size was inflated by $10 \%$ to a total of 92 participants (46 in each group). This final sample size ensured that a two-sided test with $\alpha=0.05$ would have $95 \%$ power to detect a mean difference between groups for the mental health primary outcome.

\section{Statistical analysis:}

Analyses were performed using IBM-SPSS v.22 (Chicago, IL, US). For the presentation of the participants' characteristics, data were reported as mean \pm standard deviation (SD) for those in which the assumption of normal distribution did not seem violated; otherwise, data were reported as median \pm interquartile range (IQR). Differences between groups for baseline data were evaluated by the unpaired $t$-test and the Mann-Whitney U test for continuous variables, plus Chi-square tests for categorical variables.

Data were analyzed with the intention-to-treat principle. A conservative, single-imputation approach with 'last observation carried forward' replaced missing values at followup. In our study, the baseline score replaced the missing follow-up value of an individual who did not provide followup data. Sensitivity analyses for the primary outcome were performed, including patients who completed the follow-up.

Results of the questionnaires were analyzed with the total scores and presence of a positive screening for the disorder based on cutoff values. Comparisons of positive screening between groups were performed with Chi-square tests. Data are reported as percentages (\%) and odds ratios (OR) with the $95 \%$ confidence interval (CI). For total scores, we performed nonparametric tests to compare differences between and within groups, given the baseline and follow-up values. The Mann-Whitney U test was used for between-group analyses, and the Wilcoxon signed ranks test was used for within-group analyses. Data for total scores were reported as median $\pm I Q R$. Primary outcomes used a significance level of 0.05 .

\section{Social aspects during the study}

On March 19th, 2020, the city of Porto Alegre, where most study participants reside, presented its first decree of a state of public calamity to prevent and tackle the epidemic of COVID-19. The ordinance that guides social distancing and regulates establishments was published on March 22nd, requiring social distancing for people older than 60 , as well as restrictions on the use of public squares and parks. In the following weeks, measures were tightened, restricting establishments that offer essential services under the condition of using personal protective equipment, such as masks and maintaining a minimum interpersonal distance of two meters to maintain basic activities. A teleworking regimen was always encouraged when possible. On May 11th, 2020, a strategy called "controlled distancing" was announced and put into practice in the state of Rio Grande do Sul, including Porto Alegre. This model defines structured and dynamic plans for each region based on new cases, the number of hospitalized patients, and the number of available hospital beds. Depending on the degree of risk, each region receives restrictive measures, as per local peculiarities. Despite flexibility of restrictions at some points, social distancing for risk groups, such as those with diabetes, remained high throughout the study period. During the pandemic period, the endocrinology division restructured the assistance organization in order to offer teleconsultations to patients with diabetes. The adaptation to remote assistance took place from May to June and gradually improved, reaching the peak of teleconsultations performed in the months of August and September. Thus, the usual care during the pandemic consisted of the delay of face-to-face medical appointments and the provision of telephone calls by the attending physician periodically.

\section{Results}

A total of 138 potentially eligible patients were identified, and recruitment stopped after finding the planned sample size. One patient was lost due to registry error. Despite 16 phone calls were proposed, some participants did not answer all weekly calls. The median number of phone calls received by participants in the intervention group was 15 (IRQ 14-16). Two patients received less than 10 calls, as requested (one received five calls and the other two calls). During the intervention, only two participants needed clinical support with adjustment of insulin doses, due to recurrent hypoglycemia; all other interventions were focused on mental health support and multidisciplinary strategies. At the end of the study, six patients did not respond to the final questionnaires, two patients did not answer the final phone call, one patient was hospitalized and did not want to interact with the researcher, and one patient was confused during the evaluation, unable to respond (see Fig. 1).

\section{Participant characteristics}

Overall $(n=91)$, participants had a mean age of $61.3 \pm 9.1$ years; $64.8 \%$ were female, $78.0 \%$ white, and $50.6 \%$ married. Eighty percent had lower-middle income and $30.6 \%$ had regular employment before the COVID-19 pandemic. The mean diabetes duration was $18.1 \pm 9.5$ years, and the $\mathrm{HbA} 1 \mathrm{c}$ value was $8.8 \pm 1.7 \%(73.0 \pm 18.6 \mathrm{mmol} / \mathrm{mol})$, with $83.5 \%$ of participants on insulin treatment. In general, $20.9 \%$ of participants had a previous depression diagnosis, $6.6 \%$ had anxiety diagnosis, and $2.2 \%$ had bipolar disorder diagnosis. Around $25.3 \%$ regularly used antidepressants and 
Fig. 1 Flow diagram of the study

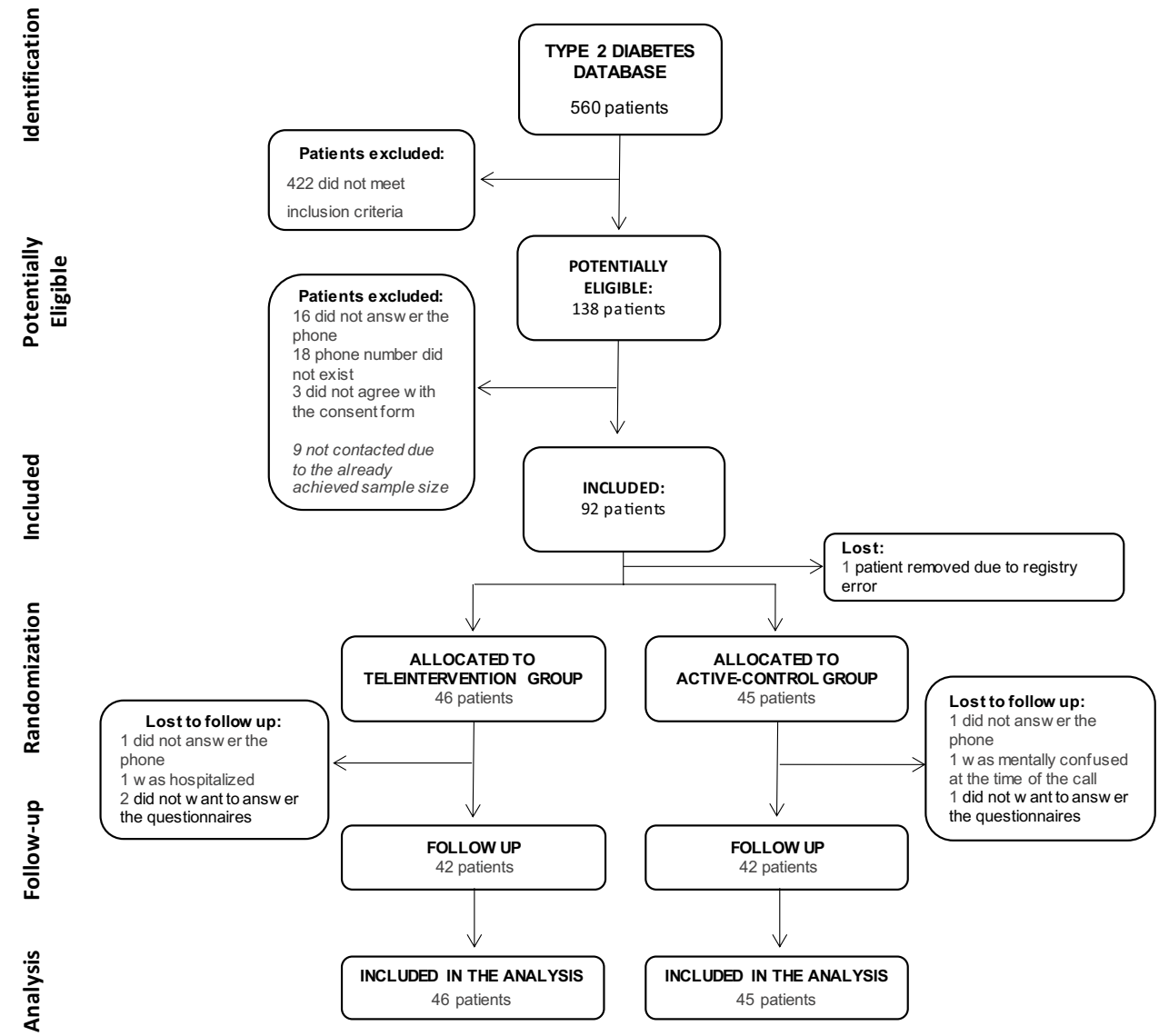

9.9\% used neuroleptic drugs. The two groups were comparable in all baseline characteristics (see Table 1).

Regarding social aspects during COVID-19, 32.9\% followed total social distancing and $57.6 \%$ followed only partially (leaving home for basic activities, such as market, pharmacy, and health care). Most participants (69.4\%) had contact with family during the study and $17.6 \%$ did not. The majority of participants $(54.1 \%)$ had reduced family income and $5.9 \%$ lost their jobs during the pandemic. Regarding clinical issues, $24.7 \%$ had respiratory symptoms during the pandemic, $5.9 \%$ had confirmed COVID-19 infection, and $7.1 \%$ required hospitalization for any reason.

\section{Primary outcome}

\section{Mental health disorders screening:}

Considering the pre-established cutoff values, a positive screening for mental health disorders was similar between groups at the time of inclusion (baseline). After 16 weeks of follow-up, positive screening was found in $37.0 \%$ of participants in the teleintervention group vs. 57.8\% of participants in the active control group $(P=0.04)$ (see Fig. 2). The active control group had a likelihood of 2.33 (95\% CI, 1.01-5.42) of presenting positive screening for mental health disorders in relation to the intervention group in the followup (see Table 3). Sensitivity analysis was performed, including only participants who completed the follow-up $(n=84)$. Of them, $36.6 \%$ in the teleintervention group and $62.5 \%$ in the active control group had a positive screening at followup $(p=0.02)$.

Given the total score of the SQR-20 questionnaire at follow-up, the teleintervention group presented a median of 5.0 (2.0-9.0) vs. 8.0 (3.0-12.0) in the active control group $(P=0.09)$ (Table 2$)$. When including participants who completed the follow-up, this difference was greater [5.0 (2.0-9.5) in the teleintervention group vs. 8.5 (3.3-12.0) in the active control group; $P=0.05]$. SRQ 20: Self Report Questionnaire-20; B-PAID: Brazilian version of the Problem Areas in Diabetes Scale; EAT 26: Eating Attitudes Test; MSQ: Mini Sleep Questionnaire.

\section{Secondary outcomes}

\section{Diabetes-related emotional distress:}

Considering cutoff values at follow-up, the presence of positive screening for diabetes-related emotional distress was found in $21.7 \%$ of participants in the teleintervention group vs. $42.2 \%$ in the active control group $(P=0.03)$ (see 
Table 1 Baseline characteristics of study participants

\begin{tabular}{|c|c|c|c|c|}
\hline & Total $(N=91)$ & $\begin{array}{l}\text { Active control } \\
\text { group }(n=45)\end{array}$ & $\begin{array}{l}\text { Teleintervention } \\
\text { group }(n=46)\end{array}$ & $P$ value \\
\hline Age (years) & $61.3 \pm 9.1$ & $61.0 \pm 9.0$ & $61.6 \pm 9.2$ & 0.76 \\
\hline Sex (\% female $)$ & $64.8 \%$ & $66.7 \%$ & $63.0 \%$ & 0.71 \\
\hline Race/ethnicity (\% white) & $78.0 \%$ & $73.3 \%$ & $82.6 \%$ & 0.29 \\
\hline Marital status (\% married) & $50.6 \%$ & $46.3 \%$ & $54.5 \%$ & 0.45 \\
\hline Lower-middle income* (\%) & $80.0 \%$ & $85.4 \%$ & $75.0 \%$ & 0.23 \\
\hline Regular employment (\%) & $30.6 \%$ & $31.7 \%$ & $29.5 \%$ & 0.82 \\
\hline \multicolumn{5}{|l|}{ Diabetes aspects } \\
\hline Diabetes duration (years) & $18.1 \pm 9.5$ & $18.7 \pm 9.0$ & $17.5 \pm 9.6$ & 0.56 \\
\hline $\mathrm{HbA} 1 \mathrm{c}(\%)(\mathrm{mmol} / \mathrm{mol})$ & $\begin{array}{l}8.8 \pm 1.7 \\
73.0 \pm 18.6\end{array}$ & $\begin{array}{l}9.0 \pm 1.6 \\
75.0 \pm 17.5\end{array}$ & $\begin{array}{l}8.5 \pm 1.7 \\
69.0 \pm 18.6\end{array}$ & 0.11 \\
\hline \multicolumn{5}{|l|}{ Diabetes complications } \\
\hline Retinopathy & $40.7 \%$ & $44.4 \%$ & $30.4 \%$ & 0.47 \\
\hline Neuropathy & $29.7 \%$ & $28.9 \%$ & $37.0 \%$ & 0.87 \\
\hline Nephropathy & $42.9 \%$ & $40.0 \%$ & $45.7 \%$ & 0.59 \\
\hline Insulin use (\%) & $83.5 \%$ & $82.2 \%$ & $84.8 \%$ & 0.74 \\
\hline Metformin use (\%) & $75.8 \%$ & $80.0 \%$ & $71.7 \%$ & 0.36 \\
\hline \multicolumn{5}{|l|}{ Previous diseases } \\
\hline Systemic arterial hypertension (\%) & $82.4 \%$ & $80.0 \%$ & $84.8 \%$ & 0.55 \\
\hline Cardiovascular disease $(\%)$ & $39.6 \%$ & $34.8 \%$ & $44.4 \%$ & 0.35 \\
\hline ACE or ARB inhibitors use (\%) & $82.4 \%$ & $80.0 \%$ & $84.8 \%$ & 0.55 \\
\hline Statins use (\%) & $82.4 \%$ & $84.4 \%$ & $80.4 \%$ & 0.62 \\
\hline ASA use $(\%)$ & $52.7 \%$ & $55.6 \%$ & $50.0 \%$ & 0.60 \\
\hline \multicolumn{5}{|l|}{ History of mental disorders } \\
\hline Depression (\%) & $20.9 \%$ & $20.0 \%$ & $21.7 \%$ & 0.84 \\
\hline Anxiety (\%) & $6.6 \%$ & $2.2 \%$ & $10.9 \%$ & 0.10 \\
\hline Bipolar disorder (\%) & $2.2 \%$ & $2.2 \%$ & $2.2 \%$ & 0.99 \\
\hline Other psychiatric conditions (\%) & $2.2 \%$ & $2.2 \%$ & $2.2 \%$ & 0.99 \\
\hline SRI use (\%) & $24.2 \%$ & $22.2 \%$ & $26.1 \%$ & 0.67 \\
\hline Tricyclic antidepressant use (\%) & $8.8 \%$ & $8.9 \%$ & $8.7 \%$ & 0.97 \\
\hline Lithium use (\%) & $2.2 \%$ & $2.2 \%$ & $2.2 \%$ & 0.99 \\
\hline Antipsychotic use (\%) & $6.6 \%$ & $4.4 \%$ & $8.7 \%$ & 0.41 \\
\hline Benzodiazepine use (\%) & $3.3 \%$ & $2.2 \%$ & $4.3 \%$ & 0.57 \\
\hline \multicolumn{5}{|l|}{ Pandemic-related aspects } \\
\hline \multicolumn{5}{|l|}{ Social distancing (self-reported) } \\
\hline $\begin{array}{l}\text { Partial } \\
\text { Total } \\
\text { None }\end{array}$ & $\begin{array}{l}57.6 \% \\
32.9 \% \\
9.4 \%\end{array}$ & $\begin{array}{l}51.2 \% \\
36.6 \% \\
12.2 \%\end{array}$ & $\begin{array}{l}63.6 \% \\
29.5 \% \\
6.8 \%\end{array}$ & 0.46 \\
\hline \multicolumn{5}{|l|}{ Social contact } \\
\hline $\begin{array}{l}\text { Only family } \\
\text { Family and friends } \\
\text { None }\end{array}$ & $\begin{array}{l}69.4 \% \\
12.9 \% \\
17.6 \%\end{array}$ & $\begin{array}{l}73.2 \% \\
14.6 \% \\
12.2 \%\end{array}$ & $\begin{array}{l}65.9 \% \\
11.4 \% \\
22.7 \%\end{array}$ & 0.43 \\
\hline Reduction in family income (\%) & $54.1 \%$ & $61.0 \%$ & $47.7 \%$ & 0.22 \\
\hline Lost the job (\%) & $5.9 \%$ & $2.4 \%$ & $9.1 \%$ & 0.19 \\
\hline Presented respiratory symptoms (\%) & $24.7 \%$ & $29.3 \%$ & $20.5 \%$ & 0.35 \\
\hline Confirmed COVID-19 infection & $5.9 \%$ & $7.3 \%$ & $4.5 \%$ & 0.59 \\
\hline Hospitalization & $7.1 \%$ & $4.9 \%$ & $9.1 \%$ & 0.45 \\
\hline
\end{tabular}

Data are mean \pm standard deviation $(\mathrm{SD})$ or $\mathrm{n}(\%)$. Plus, $\alpha \leq 0.05$ indicates significant difference. HbA1c: hemoglobin A1c; ACE: angiotensin-converting enzyme; ARB: angiotensin II receptor blocker; ASA: acetylsalicylic acid; SRI: serotonin reuptake inhibitors (includes selective serotonin reuptake inhibitors and dual serotonin/norepinephrine reuptake inhibitors). Partial social distancing includes patients who go out for basic activities: market, pharmacy, and health care. Total social distancing includes patients who follow the orientation of home-quarantine. *Family monthly income less than the equivalent of \$ 539.19 (USD) 
Fig. 2 Participants with positive screening for the proposed assessments, based on cutoff values and comparison between intervention and control groups. Legend: number of participants who present positive screening based on pre-established cutoff values. For the evaluation of mental health disorders, a score greater than or equal to 7 on SRQ 20 is considered positive. Diabetes-related emotional distress is considered when the B-PAID score is greater than or equal to 40 . The presence of positive screening for an eating disorder is considered when the EAT 26 score is greater than or equal to 20. A positive screening for sleep disorder is considered when a score greater than or equal to 31 is present in the MSQ. ${ }^{*} P=0.04$. ${ }^{*} * P=0.03$
Mental Health Disorders (SRQ 20)
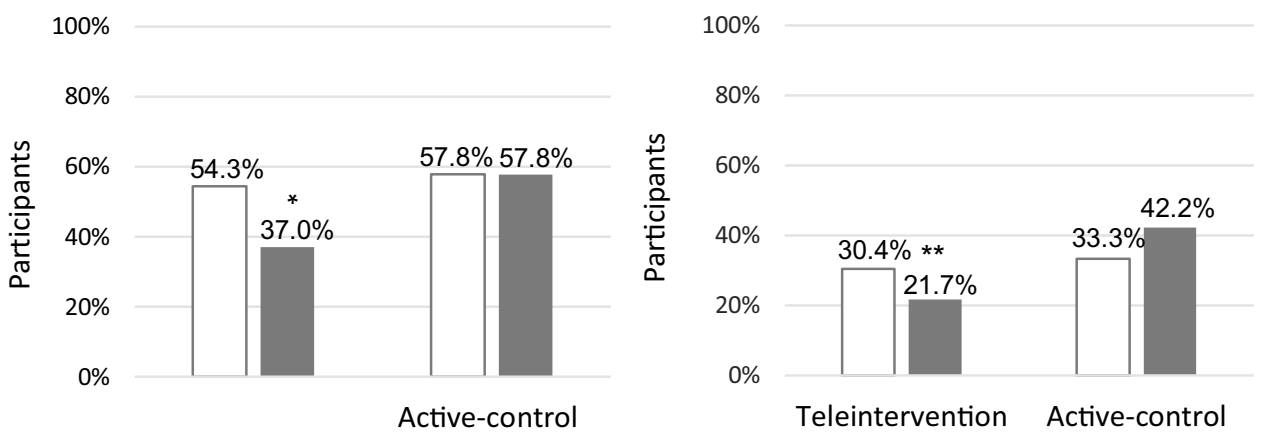

Eating Disorders (EAT 26)

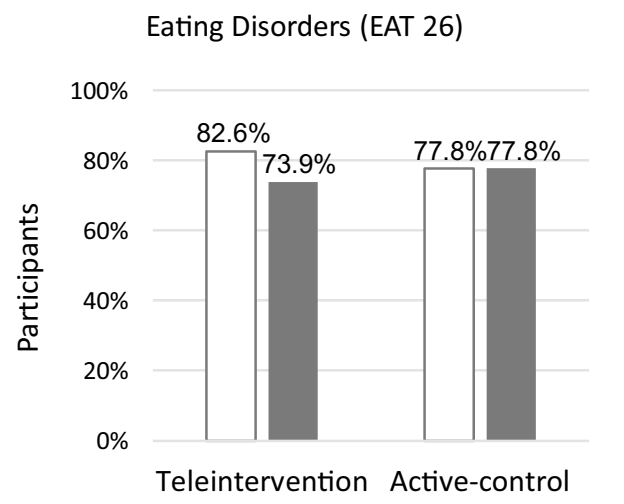

Diabetes-related Distress (B-PAID)

Teleintervention Active-control

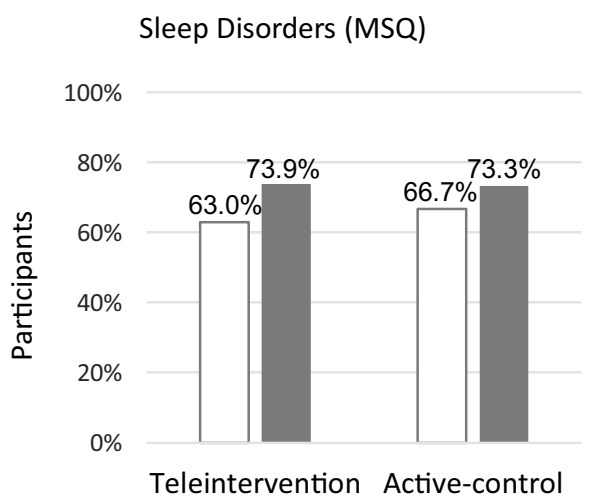

Baseline

Follow-up

Fig. 2). The control group had a likelihood of 2.63 (95\% CI, 1.05-6.58) of presenting positive screening for diabetes-related emotional distress in relation to the intervention group in the follow-up (see Table 3). Considering the B-PAID total score, groups were comparable at baseline. In the follow-up, the teleintervention group presented a median of 12.5 (6.0 to 29.5) vs. $27.0(6.0-47.5)$ in the active control group $(P=0.08)$ (see Table 2$)$.

\section{Eating disorders:}

At follow-up, the presence of positive screening for eating disorders was found in $73.9 \%$ of participants in the teleintervention group vs. $77.8 \%$ of participants in the active control group $(P=0.67)$. Considering the total score, the groups were comparable at both baseline and follow-up.

\section{Sleep disorders:}

Positive screening for sleep disorders was found in $73.9 \%$ of participants in the teleintervention group vs. $73.3 \%$ of participants in the active control group $(\mathrm{P}=0.95)$. Groups were comparable at baseline and follow-up in the MSQ total score.

\section{Treatment adherence:}

Considering the total score for treatment adherence, the groups were comparable at baseline. At follow-up, the teleintervention group presented a median of 53.0 (46.8 to 57.3) vs. $53.0(44.0-60.0)$ in the active control group, with no difference between $(P=0.73)$ or within-groups.

\section{Discussion}

This was a controlled randomized trial to assess the impact of a teleintervention on mental health parameters in patients with type 2 diabetes during the COVID-19 pandemic. After 16 weeks of follow-up, participants in the teleintervention group had a $36 \%$ lower prevalence of positive screening for mental health disorders than the control group. The proposed teleintervention reduced diabetes-related emotional distress by almost half. Despite the high prevalence of eating and sleep disorders in this period, there was no change in scores related to the proposed teleintervention.

The psychosocial repercussions of COVID-19 represent one phase of the disease with potential to generate lasting damage. Fear of illness and uncertainty about the future can 
Table 2 Comparison of questionnaires' total scores for baseline and for follow-up after 16 weeks

\begin{tabular}{|c|c|c|c|}
\hline & Active control group $(n=45)$ & Teleintervention group $(n=46)$ & $\mathrm{P}$ value \\
\hline \multicolumn{4}{|l|}{ Mental health disorders (SRQ 20) } \\
\hline Baseline & $6.0(2.5$ to 11.0$)$ & $6.0(3.0$ to 9.3$)$ & 0.76 \\
\hline Follow-up & $8.0(3.0$ to 12.0$)$ & $5.0(2.0$ to 9.0$)$ & 0.09 \\
\hline $\begin{array}{l}\text { Change in scores } \\
\text { Difference within-oroun (P value) }\end{array}$ & $0.0(-0.3$ to 1.4$)$ & $0.0(-0.3$ to 0.7$)$ & \\
\hline & 0.32 & 0.53 & \\
\hline \multicolumn{4}{|l|}{ Diabetes-related distress (B-PAID) } \\
\hline Baseline & $18.0(6.5$ to 39.0$)$ & $21.0(11.8$ to 8.0$)$ & 0.43 \\
\hline Follow-up & $27.0(6.0$ to 47.5$)$ & 12.5 (6.0 to 29.5$)$ & 0.08 \\
\hline Change in scores & $0.1(-0.6$ to 0.1$)$ & $-0.3(-0.7$ to -0.3$)$ & \\
\hline Dinterence witnm-group (F value) & 0.29 & 0.04 & \\
\hline \multicolumn{4}{|l|}{ Treatment adherence (SCI-R) } \\
\hline Baseline & $51.0(44.0$ to 57.0$)$ & $51.5(46.8$ to 6.0$)$ & 0.80 \\
\hline Follow-up & $53.0(44.0$ to 60.0$)$ & $53.0(46.8$ to 7.3$)$ & 0.73 \\
\hline $\begin{array}{l}\text { Change in scores } \\
\text { Difference within-group (P value) }\end{array}$ & $0.0(-0.2$ to 0.2$)$ & $0.0(-0.1$ to 0.2$)$ & \\
\hline & 0.54 & 0.25 & \\
\hline \multicolumn{4}{|l|}{ Eating disorders (EAT 26) } \\
\hline Baseline & $29.0(20.5$ to 32.5$)$ & $26.5(21.0$ to 1.3$)$ & 0.67 \\
\hline Follow-up & $27.0(20.0$ to 33.0$)$ & $24.5(18.8$ to 0.3$)$ & 0.50 \\
\hline $\begin{array}{l}\text { Change in scores } \\
\text { Difference within-oroun (P yalue) }\end{array}$ & $-0.1(0.3$ to -0.1$)$ & $0.0(-0.3$ to 0.3$)$ & \\
\hline Dinterence wimm-group (F value) & 0.44 & 0.58 & \\
\hline \multicolumn{4}{|l|}{ Sleep disorders $(M S Q)$} \\
\hline Baseline & $39.0(27.5$ to 48.0$)$ & $35.0(25.8$ to 6.8$)$ & 0.47 \\
\hline Follow-up & $38.0(28.0$ to 52.0$)$ & $36.0(28.0$ to 5.0$)$ & 0.35 \\
\hline $\begin{array}{l}\text { Change in scores } \\
\text { Difference within-group (P value) }\end{array}$ & $0.0(-0.2$ to 0.5$)$ & $0.0(-0.1$ to 0.4$)$ & \\
\hline & 0.55 & 0.47 & \\
\hline
\end{tabular}

Data are median and interquartile range (IQR). Also, $\alpha \leq 0.05$ indicates a significant difference. The change in scores was evaluated using the formula (follow-up score-baseline score)/baseline score. SRQ 20: Self Report Questionnaire-20; B-PAID: Brazilian version of the Problem Areas in Diabetes Scale; EAT-26: Eating Attitudes Test; MSQ: Mini Sleep Questionnaire; SCI-R: Self-Care Inventory- Revised.

Table 3 Likelihood for positive screening in the proposed assessments based on cutoff values and comparison between intervention and control groups

\begin{tabular}{lcc}
\hline & Active control group $(n=45)$ & $\begin{array}{l}\text { Teleinterven- } \\
\text { tion group } \\
(n=46)\end{array}$ \\
\hline $\begin{array}{l}\text { Mental health disorders (SRQ 20) } \\
\text { Baseline }\end{array}$ & $1.15(0.50-2.63)$ & 1 \\
Follow-up & $2.33(1.01-5.42)$ & 1 \\
Diabetes-related distress (B-PAID) & & \\
Baseline & $1.14(0.47-2.76)$ & 1 \\
Follow-up & $2.63(1.05-6.58)$ & 1 \\
Eating disorders $(\boldsymbol{E A T ~ 2 6 )}$ & & 1 \\
Baseline & $0.74(0.26-2.08)$ & 1 \\
Follow-up & $1.24(0.47-3.24)$ & 1 \\
Sleep disorders $(\mathbf{M S Q})$ & & 1 \\
Baseline & $1.17(0.49-2.78)$ & \\
Follow-up & $0.97(0.38-2.46)$ & \\
\hline
\end{tabular}

Data are odds ratio (OR) and $95 \%$ confidence interval (CI). Odds ratio $\geq 1$ represents a greater likelihood for the proposed disorder in relation to the intervention group. SRQ 20: Self Report Questionnaire-20; B-PAID: Brazilian version of the Problem Areas in Diabetes Scale; EAT-26: Eating Attitudes Test; MSQ: Mini Sleep Questionnaire 
precipitate anxiety and stress-related disorders [17]. Beyond stress inherent to the illness itself, mass home-confinement directives raised concerns about how people would react individually and collectively [18]. A recent review of psychological impact of quarantined people revealed numerous emotional outcomes, including stress, depression, irritability, insomnia, and fear, which can persist even after the quarantine ends [19]. Some groups may be more vulnerable to the psychosocial effects of pandemics [18]. Patients with diabetes have a higher prevalence of mood and anxiety disorders compared to the general population under non-pandemic conditions [4-6], so it was expected that they could be affected more significantly. A recent study published by our group showed $44.2 \%$ prevalence of minor psychiatric disorders in patients with diabetes during the COVID-19 pandemic [3]. In this study, at the end of four months of the pandemic, almost $60 \%$ of participants in the control group had a positive screening for mental health disorders, with the possibility of reducing this to $36 \%$ by maintaining regular telephone calls with health professionals. The benefits of contact reduced diabetes-related emotional distress as well. These data reinforce the importance of developing remote care strategies to mitigate the psychological effects of the COVID-19 pandemic, especially for patients with type 2 diabetes.

Despite high expectations regarding telemedicine, only few studies evaluated the benefits of remote interventions for chronic diseases during the COVID-19 pandemic. Van Dijk et al. assessed maintaining a telephone conversation program in a sample of older adults with chronic affective disorders. This study showed positive results in this group, especially in relation to the development of resilience and adaptation to the new phase [20]. Another study by Wei et al. performed an internet-based intervention for relaxation and self-care, and found that patients exhibited significantly decreased levels of depression and anxiety symptoms vs. those in the control group during the COVID-19 pandemic [21]. In patients with diabetes, studies carried out in previous years in non-pandemic situations show that remote interventions are well received and have the potential to improve psychological well-being [16, 22]. Our study found that the benefits of teleinterventions in patients with diabetes are also positive when applied in catastrophic situations. Although our intervention presented a multidisciplinary approach and complemented medical care, it should be noted that only two patients needed prescription adjustments during the intervention period. We hypothesize that its impact on mental health was mainly due to active listening and emotional support offered during the pandemic.

This study has some limitations. First, patients were selected from two tertiary care centers, which can limit external validity. Moreover, a high number of patients used insulin regularly (about $83 \%$ ), which differs from what is expected for patients with type 2 diabetes in our population and reflects a more severe and long-standing disease. A meta-analysis carried out by Bai et al. showed that patients on insulin therapy had a higher risk of depressive symptoms compared with those on oral antidiabetic drugs ( $\mathrm{OR}=1.42,95 \% \mathrm{CI} 1.08$ to 1.86$)$ [23]. Thus, it is possible that patients included in our sample were more vulnerable to developing mental health disorders. Second, the scales used to assess mental health disorders were designed for self-application. As the questionnaires were applied via telephone contact, that could be a potential source of bias. To minimize this effect, the researchers strictly followed steps of the questionnaires, repeating alternative answers to each question when requested. Moreover, the scales used to assess psychiatric disorders work as screening tools and have no diagnostic value. Third, we considered that a relatively small sample was included in this study, but still in accordance with sample size calculation. The small sample did not allow subgroup analyses to be carried out, which could be important for future studies. Fourth, considering the scarcity of studies evaluating the impact of teleinterventions in mental health outcomes on patients living with diabetes, there are no answers to date on the ideal frequency of telehealth appointments in crisis situations. Thus, it is possible that the proposed intervention model does not reflect accurately what would usually happen in pandemic situations. Even so, it has an undeniable value to guide telehealth strategies to support these patients in similar situations in the future. Fifth, considering the availability of participants' electronic devices, we opted for the use of remote contact through telephone calls in the intervention proposed. The non-use of new technologies, such as video calls and automated data capturing, may be considered a limitation of the strategy performed. Finally, the limitations related to the active control group must be mentioned. The lower availability of health care during the COVID-19 pandemic may have had a negative impact on this group. To reduce the gap between the intervention and control group during the study period, an informational website was made available for this group.

The COVID-19 pandemic has had serious repercussions on psychological and social functioning, which seems more significant for patients with diabetes. Developing strategies to mitigate this effect are urgently needed. This study demonstrated that maintaining remote connections with health professionals during the period of social distancing had the potential to reduce mental health disorders and diabetesrelated emotional distress. In addition to providing adequate care for physical demands, monitoring psychological needs and providing psychosocial support for these patients is essential. Strategies to keep patients in contact with health professionals in periods of social crisis are essential, and should be encouraged for similar situations in the future. 
Supplementary Information The online version contains supplementary material available at https://doi.org/10.1007/s00592-021-01690-1.

Acknowledgments This work was conducted with support from Hospital de Clínicas de Porto Alegre, Postgraduate Program in Endocrinology from Universidade Federal do Rio Grande do Sul, School of Medicine from Pontifícia Universidade Católica do Rio Grande do Sul and Division of Internal Medicine from Hospital São Lucas da Pontifícia Universidade Católica do Rio Grande do Sul.

Authors Contribution JA was involved in conceptualization, methodology, software, data curation, writing - original draft preparation. GBO, DWF, GLK, ASB, BBA, CPK, AB and GHT were involved in methodology and investigation. BDS was involved in conceptualization, validation, supervision, writing-Reviewing and editing. GHT was involved in conceptualization, data curation, writing - original draft preparation and supervision. JA is the guarantor of this work and, as such, had full access to all the data in the study and takes responsibility for the integrity of the data and the accuracy of the data analysis.

Funding This work was supported by FIPE (Fundo de Incentivo à Pesquisa e Eventos) of Hospital de Clínicas de Porto Alegre, and Postgraduate Program in Endocrinology from Universidade Federal do Rio Grande do Sul. The funders had no role in study design, data collection and analysis, decision to publish, or preparation of the manuscript.

\section{Declaration}

Conflict interest There is no conflict of interest to declare.

Availability of data and materials The data collected for the study, including deidentified participant data and informed consent form, will be available for one year after publication of the article upon justified request to the e-mail address of the main researcher and with a signed data access agreement

Ethics approval The study was approved by the National Research Ethics Commission of Brazil (CONEP), number 4.029.368.

Consent to participate All participants agreed to the free and informed consent form, which was saved by voice record and electronic registration.

Consent for publication All authors have reviewed the final version of the manuscript and agree with the publication of the results presented.

\section{References}

1. Huang C, Wang Y, Li X et al (2020) Clinical features of patients infected with 2019 novel coronavirus in Wuhan. Lancet, China. https://doi.org/10.1016/S0140-6736(20)30183-5

2. Jin ZJ, Dong X, Yuan CY et al (2020) Clinical characteristics of 140 patients infected with SARS-CoV-2 in Wuhan, China. Allergy Eur J Allergy Clin Immunol. https://doi.org/10.1111/all.14238

3. Alessi J, De Oliveira GB, Franco DW et al (2020) Mental health in the era of COVID-19: Prevalence of psychiatric disorders in a cohort of patients with type 1 and type 2 diabetes during the social distancing. Diabetol Metab Syndr. https://doi.org/10.1186/ s13098-020-00584-6

4. Meurs M, Roest AM, Wolffenbuttel BHR et al (2016) Association of depressive and anxiety disorders with diagnosed versus undiagnosed diabetes: An epidemiological study of 90,686 participants. Psychosom Med. https://doi.org/10.1097/PSY.00000 00000000255

5. Mezuk B, Eaton WW, Albrecht S, Golden SH (2008) Depression and type 2 diabetes over the lifespan: A meta-analysis. Diabetes Care. https://doi.org/10.2337/dc08-0985

6. Smith KJ, Béland M, Clyde M et al (2013) Association of diabetes with anxiety: A systematic review and meta-analysis. J Psychosom Res 74(2):89-99

7. Jung SJ, Jun JY (2020) Mental health and psychological intervention amid COVID-19 outbreak: Perspectives from South Korea. Yonsei Med J 61(4):271

8. Lima CKT, de Carvalho Medeiros PM, de Lima IAAS et al (2020) The emotional impact of Coronavirus 2019-nCoV (new Coronavirus disease). Psychiatry Res 287:112915

9. Idris H (2020) Utilization of teleconsultation: Mitigation in handling mental disorders in the COVID-19 era. Int J Ment Health Addict. https://doi.org/10.1007/s11469-020-00323-y

10. Rennie D (2001) CONSORT Revised-Improving the reporting of randomized trials. J Am Med Assoc 285(15):2006-2007

11. Gonçalves DM, Stein AT, Kapczinski F (2008) Avaliação de desempenho do Self-Reporting Questionnaire como instrumento de rastreamento psiquiátrico: Um estudo comparativo com o Structured Clinical Interview for DSM-IV-TR. Cad Saude Publica. https://doi.org/10.1590/S0102-311X2008000200017

12. Gross CC, Scain SF, Scheffel R, Gross JL, Hutz CS (2007) Brazilian version of the Problem Areas in Diabetes Scale (B-PAID): Validation and identification of individuals at high risk for emotional distress. Diabetes Res Clin Pract 76:455-459. https://doi. org/10.1016/j.diabres.2006.09.022

13. Bighetti F, Dos Santos CB, Dos Santos JE, Ribeiro RPP (2004) Tradução e validação do Eating Attitudes Test em adolescentes do sexo feminino de Ribeirão Preto, São Paulo. J Bras Psiquiatr

14. Falavigna A, De Souza Bezerra ML, Teles AR et al (2011) Consistency and reliability of the Brazilian Portuguese version of the Mini-Sleep Questionnaire in undergraduate students. Sleep Breath. https://doi.org/10.1007/s11325-010-0392-x

15. Teló GH, de Iorra FQ, Velho BS et al (2020) Validation to brazilian portuguese of the self-care inventory-revised for adults with type 2 diabetes. Arch Endocrinol Metab 64(2):190-194

16. Pouwer F, Snoek FJ, Van Der Ploeg HM et al (2001) Monitoring of psychological well-being in outpatients with diabetes: Effects on mood, HbA1c, and the patient's evaluation of the quality of diabetes care: A randomized controlled trial. Diabetes Care. https://doi.org/10.2337/diacare.24.11.1929

17. Troyer EA, Kohn JN, Hong S (2020) Are we facing a crashing wave of neuropsychiatric sequelae of COVID-19? Neuropsychiatric symptoms and potential immunologic mechanisms. Brain Behav Immun 87:34-39. https://doi.org/10.1016/j.bbi.2020.04. 027

18. Pfefferbaum B, North CS (2020) Mental Health and the Covid-19 Pandemic. N Engl J Med. https://doi.org/10.1056/nejmp2008017

19. Brooks SK, Webster RK, Smith LE et al (2020) The psychological impact of quarantine and how to reduce it: rapid review of the evidence. Lancet 395(10227):912-920

20. van Dijk SDM, Bouman R, Folmer EH et al (2020) (Vi)-rushed Into Online Group Schema Therapy Based Day-Treatment for Older Adults by the COVID-19 Outbreak in the Netherlands. Am J Geriatr Psychiatry. https://doi.org/10.1016/j.jagp.2020.05.028

21. Wei N, Huang BC, Lu SJ, Hu JB, Zhou XY, Hu CC, Chen JK, Huang JW, Li SG, Wang Z, Wang DD, Xu Y, Hu SH (2020) Efficacy of internet-based integrated intervention on depression and anxiety symptoms in patients with COVID-19. J Zhejiang Univ Sci B 21(5):400-404. https://doi.org/10.1631/jzus.B2010013

22. Long AF, Gambling T, Young RJ et al (2005) Acceptability and satisfaction with a telecarer approach to the management of type 
2 diabetes. Diabetes Care. https://doi.org/10.2337/diacare.28.2. 283

23. Bai X, Liu Z, Li Z, Yan D (2018) The association between insulin therapy and depression in patients with type 2 diabetes mellitus: A meta-analysis. BMJ Open. https://doi.org/10.1136/bmjop en-2017-020062
Publisher's Note Springer Nature remains neutral with regard to jurisdictional claims in published maps and institutional affiliations.

\section{Authors and Affiliations}

\section{Janine Alessi ${ }^{1,2}$ (1) . Giovana Berger de Oliveira ${ }^{3} \cdot$ Debora Wilke Franco $^{3} \cdot$ Alice Scalzilli Becker $^{3}$. Carolina Padilla Knijnik ${ }^{3}$. Gabriel Luiz Kobe ${ }^{3}$. Bibiana Brino Amaral ${ }^{3}$ - Ariane de Brito ${ }^{1}$ - Beatriz D. Schaan ${ }^{1,4,5}$. Gabriela Heiden Telo ${ }^{2,3,6}$}

1 Postgraduate Program in Endocrinology, Universidade Federal Do Rio Grande Do Sul, Rua Ramiro Barcelos, $\mathrm{n}^{\circ}$ 2400, $2^{\circ}$ andar, Porto Alegre, RS 90035-003, Brazil

2 Division of Internal Medicine, Hospital São Lucas da Pontifícia Universidade Católica Do Rio Grande Do Sul, Av. Ipiranga, $\mathrm{n}^{\circ}$ 6690, $6^{\circ}$ andar, Porto Alegre, RS 90160-092, Brazil

3 School of Medicine, Pontifícia Universidade Católica Do Rio Grande Do Sul, Av. Ipiranga, $n^{\circ}$ 6681, prédio 12A, Porto Alegre, RS 90160-092, Brazil
4 School of Medicine, Universidade Federal do Rio Grande do Sul, Rua Ramiro Barcelos, $n^{\circ} 2400,3^{\circ}$ andar, Porto Alegre, RS 90035-003, Brazil

5 Endocrinology Division, Hospital de Clínicas de Porto Alegre, Rua Ramiro Barcelos, 2350, prédio 12, $4^{\circ}$ andar, Porto Alegre, RS 90035-003, Brazil

6 Medicine and Health Sciences Program, Pontifícia Universidade Católica da PUCRS, Av. Ipiranga, nº 6681, prédio 12A, Porto Alegre, RS 90160-092, Brazil 